\title{
Does Gravitation Have an Influence on Electromagnetism?
}

\author{
Guido Zbiral \\ Konradtgasse 34, 3400 Klosterneuburg, Austria \\ Email: guido@zbiral.at
}

Received June 15, 2012; revised July 25, 2012; accepted August 1, 2012

\begin{abstract}
For many years physicists have been engaged on research around the globe in fields such as the unification of gravitation and electromagnetism, and an explanation for dark matter and dark energy, etc., but so far to little avail. One is left with the impression that something might be fundamentally wrong with the premises underlying the doctrine of physics applicable today, which is preventing a solution of these problems from being found. As a possible cause, the author proposes that the gravitation of the photons is not so negligible that it can be completely ignored (although this assumption does not accord with the current state of physics). Departing therefore from the accepted doctrine, he assumes that gravitation might possess a hitherto unknown important influence on electromagnetism. This paper then examines the consequences of this assumption on physics. A precise analysis will lead to the insight that the gravitation of a photon is as dynamic as the photon itself, and therefore must be taken into account with all associated physical considerations. The hitherto accepted case of a static gravitation of photons, on the other hand, can be totally neglected, as it does not exist for photons. Of key importance is the statement that the gravitation of photons is produced by gravitational quanta, and thus appears in quantised form. It is therefore necessary to rethink the physics of photons. This leads to a number of other interesting insights, as will be borne out in the further course of this paper. In the event that the assumption of the influence of gravitation on electromagnetism turns out to be correct, then this would represent a major step in unravelling the still largely unknown nature of gravitation and its significance in the natural events of the microcosmos; furtheron it would be an important contribution regarding a "New Physics" and a "New Cosmology".
\end{abstract}

Keywords: Photon; Gravitation; Gravitational Quanta; Speed of Light; Maxwell’s Theory

\section{Introduction}

Around the globe, physicists have long been engaged in research—albeit with little success—in the following special fields of physics:

Unifying the theories of General Relativity and Quantum Theory;

The nature of dark matter and dark energy;

The discovery of gravitons and evidence for the existence of cosmic gravitational waves.

All this gives the impression that at least one of the assumptions, which underlie either the current doctrine in physics or today's physical and/or cosmological model conceptions, must be wrong or incomplete-otherwise one or the other breakthroughs in these fields of physics would already have taken place.

I am convinced that a "radically new physical approach" is necessary, in order to introduce some movement into this current state of stagnation. It is clear that this new approach cannot completely accord with the conventional doctrine, otherwise everything would remain unchanged. A deliberately new way of thinking is called for. This does not represent a faulty way of thinking, but is a tactical necessity. I have therefore selected the following strategy for this paper:

I shall make an assumption, which is in contradiction with today's accepted doctrine in physics, but in my opinion is best suited for discovering new physical relationships:

1) I shall assume that there exists a hitherto unknown close relationship between gravitation and electromagnetism;

2) This assumption must be fully justified by physical arguments, which comply with today's doctrine;

3) In conclusion it will be determined, what the consequences of this assumption are for today's physics-in particular, whether it may result in new insights.

\section{Arguments Pointing to a Possible Close Relationship between Gravitation and Electromagnetism}

Remarks concerning static and dynamic gravitation:

Following the original creation of the cosmos consist- 
ing only of radiation energy (electromagnetic primordial photon radiation and superstrong gravitational radiation), no "static gravitation" could have existed in the "Big Bang" phase or shortly afterwards. In the quantum era, gravitation was to an overwhelming extent dynamic in nature and can therefore only have been created by means of gravitational quanta! The photons' dynamic gravitation remains conserved for the total lifetime of the photons, which are the oldest and longest elementary particles in existence. Their gravitation cannot be inherently static in nature.

On the other hand, the gravitation of the baryonic elementary particles, which were only subsequently created from the highly energetic, dynamic radiation energy, as well as the gravitation of the non-baryonic "dark matter", is indeed static in nature.

The physical processes occurring in the earliest phase of the cosmos during the quantum era, are even today still taking place in the photons (in qualitative terms), representing virtually a "remnant left over from that quantum era".

\section{Now to the Individual Arguments}

It is possible to derive a possible physical relationship between the speed of light (c) and the Gravitational Constant $(G)$ quite simply from $G$ itself:

Taking the gravitational constant as $\mathrm{G}=6.67 \times 10^{-11}$ $\left(\mathrm{N} \cdot \mathrm{m}^{2} \cdot \mathrm{kg}^{-2}\right)$ in consideration as well as the fact that $1 \mathrm{~N}$ is equal to $1\left(\mathrm{~kg} \cdot \mathrm{m} \cdot \mathrm{s}^{-2}\right)$, then a physical dimension for $\mathrm{G}$ of $\left(\mathrm{m}^{3} \cdot \mathrm{kg}^{-1} \cdot \mathrm{s}^{-2}\right)$ is arrived at. One can see, that the physical dimension of $\mathrm{c}^{2}\left(\mathrm{~m}^{2} \cdot \mathrm{s}^{-2}\right)$ is contained in this dimension of G!

Thus the gravitational constant itself certainly has something in common with the speed of light. Without changing any of the contents, it is possible to convert the gravitational constant $\mathrm{G}$ by extrapolating $\mathrm{c}^{2}$ :

$\mathrm{G}=\mathrm{c}^{2} \times 742 \times 10^{-28}\left(\mathrm{~m} \cdot \mathrm{kg}^{-1}\right)=\mathrm{c}^{2} \times$ const. or $\mathrm{G} / \mathrm{c}^{2}=7.42 \times 10^{-28}\left(\mathrm{~m} \cdot \mathrm{kg}^{-1}\right)=$ const.

This mathematical conversion merely represents an alternative notation of the gravitational constant $G$ and therefore must also be physically valid.

Possible consequence: The possibility of the constancy of the speed of light being dependent on the constancy of the gravitational constant $\mathrm{G}$ cannot therefore be excluded. Any change in the numerical value of $G$ would in this case also imply a change in the numerical value of $\mathrm{c}^{2}$, in order to maintain the constancy of the ratio $\mathrm{G} / \mathrm{c}^{2}$. As long as $\mathrm{G}$ remains a constant value, then c also remains constant and the constancy of the speed of light remains unchanged in the entire cosmos. Nevertheless, according to Special Relativity this interpretation is not permitted, because c would not then be an "independent" universal constant of nature, but dependent on $\mathrm{G}$ and therefore not a "genuine" constant of nature. According to this, the speed of light is only constant if $\mathrm{G}$ is also constant!

The expression $\mathrm{G} / \mathrm{c}^{2}=$ const., on the other hand, could well be a "genuine" constant of nature, as it occurs several times in physics, e.g. in association with the "Schwarzschild Radius” $\mathrm{R}_{\mathrm{s}}=2 \mathrm{MG} / \mathrm{c}^{2}$.

Moreover, the (for me hitherto unknown) relationship

$$
G / c^{2}=\mathrm{l}_{\text {Planck }} / \mathrm{m}_{\text {Planck }}=\text { const. }(\mathrm{m} / \mathrm{kg})
$$

also applies, which similarly reinforces my notion that $\mathrm{G} / \mathrm{c}^{2}=$ const. is an "original united constant of nature" that had applied as far back as the quantum era. Therefore for that quantum era, the above equation then becomes

$$
\mathrm{G}_{\text {super }} / \mathrm{c}_{\text {super }}^{2}=\text { const. }
$$

Since in the quantum era during the state of "supergravitation" $\left(G_{\text {super }}\right)$, the value of $G_{\text {super }}$ was many orders of magnitude higher than $\mathrm{G}$, then the speed of light ( $\mathrm{c}_{\text {super }}$ ) at that time must also have been many orders of magnitude higher than c; $\mathrm{c}_{\text {super }}$ could then explain the super rapid expansion behaviour of the very early cosmos, as in this situation the extremely hot and dense cosmic radiation energy would have expanded explosively with a speed of $c_{\text {super }}$ in the neighbouring cosmic space.

In this manner, we would have a physical justification for the super rapid expansion of the cosmos in its very early life-in contrast to today's usual, albeit completely arbitrarily constructed hypothesis of an exponentially generated "inflationary expansion".

The physical facts of the constancy of the speed of light independent of the energy of the photons as well as their wave propagation, are described in the Maxwell equations (refer to the internet link http://www.mahag.com/srt/maxwell.php, respectively http://hyperphysics.phy-astr.gsu.edu/hbase/electric/maxe q.html), which are considered one of the most solid physical theories ever founded. However, Maxwell's equations cannot provide any statement concerning the reason for the constancy of the speed of light. There is also no directly recognisable connection to gravitation, as the gravitational constant $\mathrm{G}$ does not explicitly occur in them. According to this, it would seem that electromagnetism and the photons do not have anything to do with gravitation, as confirmed by today's accepted doctrine in physics. However, it is unexpectedly possible to establish a relationship between Maxwell's theory and gravitation by means of the following "detour":

As according to Maxwell's so called Fifth Equation, the following physical relationship between the electrical field constant $\varepsilon_{0}$, the magnetic field constant $\mu_{0}$ and the speed of light $c$ exists:

$\varepsilon_{0} \cdot m_{0}=1 / c^{2}$, then $\mathrm{G} / \mathrm{c}^{2}=$ const. as well as $\mathrm{G} \cdot \varepsilon_{0} \cdot \mu_{0}$. $=$ const. can be written. This means that within Maxwell's Theory, not only the speed of light, but with it also elec- 
tromagnetism itself, is linked via its field constants $\varepsilon_{0}, \mu_{0}$ with the gravitational constant G. Are we to understand this merely as a random coincidence lacking any physical significance, or does this relationship in fact represent a very concrete relationship between these two forces of nature, as will be shown several times in the subsequent text? With regard to the relationships stated above, the expression $\mathrm{G} / \mathrm{c}^{2}=$ const. may possess a far greater physical significance than hitherto assumed.

Gravitation is known to be the weakest of the four elementary forces in the universe. Therefore the (static) gravitational force within an atomic structure between a proton and an electron is weaker than the electromagnetic force acting between them by a factor of $10^{-39}$. For this reason, the gravitational force of the baryonic components of an atom among themselves within an atom can be completely ignored compared to the electromagnetic force between the proton and electron.

This fact forms the basis for the assumption of physicists that the gravitational force of a photon, compared to its far stronger electromagnetic force, can also be completely ignored, whereby always a static gravitation is assumed (but without using the word "static", since no dynamic gravitation is so far known).

Remark: The possibility of dynamic gravitation in the form of high-frequency gravitational radiation for photons has not yet been considered, because taking gravity into account in relation to the "theory of special relativity" was never a subject of discussion. According to the "theory of general relativity", only low-frequency gravitational waves in the region of approx. ca. $10^{-1}$ to $10^{4} \mathrm{~Hz}$ are expected today in connection with spectacular cosmic events, although it has not yet been possible to prove their existence.

The current school of thought in physics is therefore based on the assumption that (static) gravitation is also not a factor to be considered with photons or the speed of light and may be completely ignored, but: Static gravitation does not exist for photons!

The gravitation of the photons cannot be of a static nature; it must be as dynamic as the photons themselves. This opens up a completely new insight into the physics of photons!

In my opinion, the assumption underlying the current doctrine of a negligible gravitation of photons is an erroneous assumption!

The gravitation of a photon experiences the same speed or dynamic as the photon itself. Therefore the gravitation of a photon must appear like a high-frequency gravitational radiation and resonate with the same frequency as the electromagnetic radiation (it is, as it were, "clocked" by the latter). It remains associated with the photon until the photon is expended or transformed by performing work. It cannot rush away in order to become "externally" noticed; it only has a specific reaction on "its own" photon and as far as the outside world is concerned, it does not exist.

Furthermore, non-baryonic relativistic mass relationships exist at the speed of light for "free" photons as is the case for baryonic elementary particles "bound" by strong forces within the atom. These two completely different initial or environmental conditions are therefore not comparable! In contrast to the school of thought in physics stated above, I therefore hold the view that dynamic gravitation in the form of gravitational radiation must definitely be taken into account with photons, particularly as still further cosmological and physical connections exist between electromagnetism and gravitation:

Preliminary remarks on the next point, which refer to the cosmos as a whole:

"A general consequence of Einstein's Theory of Gravitation (General Relativity) is that in a closed universe the total energy is always zero" [2]. "Positive energy", whose essence is in the expansion (electromagnetism), and "negative energy", whose essence is in the implosion or holding together (gravity), mutually cancel each other out exactly in a physically closed system. These two opposite forces of nature are equally strong in a physically closed system, therefore a stable state of equilibrium exists between them and the total energy is zero. The cosmos as a whole is a physically closed system. Thus in the Big Bang the sentence is not violated by the conservation of energy.

At the same time (here we mean within the first Planck time of $10^{-43}$ secs) as the appearance of the expansionary radiation energy (of the primordial photon radiation) in the Big Bang, gravity also appeared in order to act as its "stabilising counter-force". As the contribution of gravity to the total energy according to Einstein is negative, the sum of the two components is then zero. From this it follows necessarily, that:

At no time did or does energy exist without gravitation; therefore, exactly as is the case with the primordial photon radiation, all its subsequent radiations and energy forms must be affected by gravitation!!!

Thus right at the beginning of the history of cosmic creation there exists a close connection between the primordial electrodynamic photon radiation and its dynamic gravitation e.g. gravitational radiation, whereby the two dynamic forces are of equal strength, but have an opposite effect on each other. Had gravity not appeared within the first Planck time as cosmic energy, but only a short time later, then the total positive energy would have immediately fizzled out without having any effect and the cosmos would not have come about.

Preliminary remarks on point 2.5, which refer to each individual photon:

The above sentence can also be applied to each indi- 
vidual photon as the elementary fundamental component of the entire cosmos. Because the speed of light is constant, the forces influencing the photon must exist in equilibrium, with the result that no overall force influences the photon, which would otherwise accelerate or decelerate. At the speed of light the total energy of a photon is zero, therefore in this respect each photon corresponds to a "physically closed system".

The positive electromagnetic energy and the negative gravitational energy of each individual Photon are of equal strength and cancel each other out; a stable "equilibrium" exists between the two forces. There is no way for this equilibrium and hence the constancy of the speed of light to be achieved except by the dynamic gravitation of the photon. Electromagnetism is, on its own (without dynamic gravity), unable to produce a stable equilibrium. This is the evidence that each photon as a quantum of electromagnetic energy is connected with an equivalent quantum of gravitational energy.

The photon requires the full amount of its gravitational energy for its stabilisation, so there is nothing left which could penetrate to "the outside". Therefore it was erroneously assumed that photons have nothing to do with gravity. Only when the photon's electromagnetic energy has been expended or converted by performing work, does its gravitational energy appear "externally", albeit as a weak static gravitational force (a factor of $10^{-39}$ ), as the dynamic drive provided by the photon has been lost.

Furthermore, included in the established state of knowledge in physics is the fact that each quantum-no matter how small—and each form of energy (e.g. mass) have a gravitational effect themselves (i.e., they are "subject to gravity"), and therefore react to an external gravitational field. Since this also applies to photons, then they will be deflected when passing through a gravitational field, either gaining or losing energy depending on whether they are approaching or leaving the gravitational field-corresponding to a blue or red shift of a ray of light. There therefore exists an interaction between an external static gravitational field and the photon radiation passing through it.

All six criteria (taking each one into consideration separately) correspond to the certain state of knowledge in physics and therefore allow the conclusion to be made that the speed of light certainly does have something to do with the gravitational energy of photons. This very new physical insight also represents the "key fundamental prerequisite" for the explanation of dark matter.

\section{Further Effects of Gravitation on Electromagnetism}

Explanations of the terms used in the following section:

The "quantum of gravitation" is always closely associated with its related photon; it adheres, as it were, to the photon, or manifests itself with its photon as well as interacting with it.

This hitherto unknown "gravitation quantum" might therefore turn out to be the elementary particle, which imparts the phenomenon of gravitation and gravitational mass (in the event that the "Higgs mechanism" does not work as expected, particularly in connection with relativistic mass of photons).

The gravitation inherent in every elementary particle ("intrinsic gravitation") always relates in a specific manner to individual elementary particles including photons, and is, in each case, the gravitation corresponding to the elementary particle's energy state, caused by its "gravitation quantum". No form of energy exists, which is not associated with the phenomenon of gravitation.

The "graviton", on the other hand, is a term reserved for the hypothetical (not yet proven) independent boson in a future quantum theory of gravitation and is the means of the gravitational interaction between elementary particles.

From the six arguments made above, it follows that each photon must have emerged as a quantum of electromagnetic energy from the Big Bang with its quite specific (photon energy equivalent) gravitational quantum, with which it appears together and which causes the dynamic gravitation of the photon as well as its relativistic mass. What else could produce the photon's dynamic gravitation or relativistic mass? This gravitational quantum also appears due to its intimate meshing with the photon or electromagnetic photon radiation like radiation itself, namely as specific high-frequency gravitational radiation bound to the photon, whose speed of propagation is also equal to the speed of light.

A photon without dynamic gravitation does not exist. Therefore each photon consists of two components linked to each other, one expansive electromagnetic component and one gravitational radiation component acting in an opposite manner. These two physical quantities acting in opposite manner are ideally united in the photon. Therefore there must exist exactly the same number of gravitational quanta of the photons as there are of photons themselves, but their existence has not yet been noticed! This is why gravity is even today mostly still a mystery.

The hitherto unknown or unnoticed gravitational quantum therefore corresponds-as it were- to a "gravitational charge" of each single elementary particle, which for an agglomeration of elementary particles accordingly accumulates and produces a gravitational field, which cannot be compensated (in analogy to an electrical charge producing an electric field, which of course can be compensated).

The energy of photons is known to increase proportionally with its frequency $v$ in accordance with Planck's equation $E=h \cdot v$, where $h$ is Planck's constant or "effect- 
quantum”, a universal constant of nature. As electromagnetic radiation encompasses an extremely broad frequency spectrum (a ratio of up to $10^{0}-10^{25}$ ), the energy of the photons also occupies this very wide range. Not-withstanding this, all photons always travel in a vacuum with the same constant speed of light, irrespective of the energy quantum that they transport with them. Each photon requires its entire positive electromagnetic energy for the purpose of maintaining the speed of light against the negative stabilising opposing force of gravitation.

The photon travelling at the speed of light does not have "free energy" available for any additional "external effect". The only external effect it manifests is the constancy of the speed of light.

Therefore the constant speed of light must be associated with the wide-ranging variable relativistic mass of photons in such a manner that the photon rich in energy must be subject to the stabilising opposing force of gravity to a greater extent than the photon low in energy.

As already determined in the above text, the constant speed of light can only represent a state of equilibrium between the expansive force of electromagnetic radiation imparted by the photon and the opposing force of gravitation, in each case. The greater the energy of the photons, the greater is the stabilising opposing force of gravitation.

The speed of light c in a vacuum is therefore constant $299,792,458 \mathrm{~m} / \mathrm{secs}$, since this is exactly the value, at which a stable state of equilibrium is established between the dynamic gravitation of the photons and their expansive electromagnetic force, thus cancelling out the two opposing forces. Why the state of equilibrium should be achieved precisely by this value for the speed of light, depends in connection with $\mathrm{G} / \mathrm{c}^{2}=$ const. on the value of the Gavitational Constant " $G$ ".

\section{New Aspects Concerning Physical Events inside Photons}

\subsection{Regulation Process within Photons}

What happens inside the photons, i.e., what physical process must take place, in order for the speed of light to be constant irrespective of the energy of the photons, does not emerge from the Maxwell equations and has presumably not hitherto been investigated. These physical processes taking place inside the photons, which should be considered as the possible reason for the constancy of the speed of light, are dealt with below. The constancy of the speed of light itself is thus an effect of these internal processes.

After careful consideration the impression emerges that the speed of light is regulated to the constant value of c by a hitherto unnoticed "influencing factor" located within each individual photon. There is no other way to explain the constancy of the speed of light, which does not take into account of the intensity of the energy of its photons. There is obviously a typical regulation process here at work on the photons' constant velocity of propagation, which raises the following questions:

How does a "physical regulatory mechanism" work, acting on the extremely wide-ranging energy of photons in such a manner that results in a constant speed of light for all photons?

In particular, it is necessary to clarify whether there really is "an influencing factor" acting inside the photons, and if so, what its nature is, and how the regulation process of a constant speed of light actually functions. That is one of the important objectives of this paper.

As a "regulator" for the constancy of the speed of light the only candidate is thus the intrinsic gravitation of the photons themselves, since any change in energy of a photon simultaneously causes an equal change in its intrinsic gravitation as well as its relativistic mass. This change in intrinsic gravitation affects a photon to the extent that it is precisely the stabilising effect of gravitation corresponding to the existing energy state that is available out of all the energy states possible. Therefore the speed of light remains constant for all possible energy situations of a photon. There is no other possibility to act in a "stabilising" manner on a photon than by this standard physical process.

This feedback of a photon's stabilising intrinsic gravitation on its expansive electromagnetic force is the only identifiable physical cause for the constancy of the speed of light!

Note repeated here again: Both the previous and the following considerations only apply under the assumption that the speed of light is not an independent constant of nature, but is dependent on the value of " $G$ ". If it is true, however, that the speed of light actually is an independent constant of nature, as insisted upon by an essential foundation of today's modern physics, then neither a stabilising effect on the photons nor an internal control process is required in order to effect the constancy of the speed of light-in this case it is set at a fixed value predetermined by its origin.

A photon's gravitation is a dynamic field just as much as its electromagnetic field, since it exhibits the characteristic of travelling together with the photon at the speed of light. In order for a state of equilibrium to result from the two dynamic forces acting on the photon, as required from the argument above, the gravitational field must exhibit behaviour precisely opposite to that of the electromagnetic field.

Therefore the physical principles of both fields must be in every respect completely equivalent but opposed to each other. It therefore follows that the same vectorial Maxwellian theory also underlies the gravitational radia- 
tion associated with the photon as the electromagnetic radiation, i.e. they also possess a common theoretical basis!

Remark: It is highly remarkable, that the dynamic gravitation of the photons are described by the same theory as electrodynamics and electromagnetism, i.e., that a close "family relationship" exists between the two physical forces. On this basis, it can be concluded that the same family relationship also exists between static gravitation and electrostatics, since they are "descended" in each case from their dynamic quantities, as reflected in the similar structure of their formulae for static forces.

In addition, Prof. K. O. Greulich [3] in a lecture on the occasion of the Congress of the German Physical Society in 2012 in Göttingen also establishes that electrostatics and (static) gravitation are alike from a physical perspective!

Following the same logic, the gravitational radiation must possess transversal characteristics exactly as the electromagnetic radiation does, and it must also exhibit the same frequency as the electromagnetic radiation, as is indeed the case. It is only as a consequence of these conditions that there exists no resulting force on the photon, which could cause it to accelerate or decelerate. Therefore the photon travels in a completely uniform manner at the constant speed of light.

However, if the value of the gravitational constant $G$ were different to the current value, then the effect of the photons' intrinsic gravitation would also differ from the current effect and the state of equilibrium between both natural forces and hence the constant value of the speed of light would also differ from the current value. This was, in fact, the case in the stage of "super gravitation" ( $\mathrm{G}=\mathrm{G}_{\text {super }}$ ) and had, as a consequence, a value of the speed of light $c_{\text {super }}$ many orders of magnitude greater than the current value.

The constancy of the speed of light is thus the result of an extremely finely-tuned interaction between the photons' intrinsic gravitation and their electromagnetism, from which it follows that at the speed of light there exists an extremely close association between these two fundamental forces of nature:

On one hand, the photons' intrinsic gravitation limits the speed of expansion of electromagnetic energy (by establishing the state of equilibrium between the two forces of nature) - and thus determining the value of the speed of light, while, on the other hand, maintaining it at this constant value!

The hitherto unknown precise "fine tuning” between the two opposite fundamental forces of nature within photons, to which we owe the whole of creation, is one of the greatest physical miracles of nature and has remained up to now beyond human imagination. It must be very difficult-as in numerous other cases-just to be- lieve in a random coincidence!

\subsection{The Quantum Structure of Gravitation inside Photons}

Since the energy of the electromagnetic radiation is proportional to its frequency, it is necessary that the energy of the high-frequency gravitational radiation associated with a photon is also proportional to its frequency, whereby both frequencies are identical. This is necessary to achieve a stable state of equilibrium at the speed of light between the two physical quantities, which oppose each other by nature.

Therefore Planck's equation for the energy of the electromagnetic radiation of a photon $E=h \cdot v$ must also apply to the high-frequency gravitational radiation, which is associated with the photon and only selectively acts on it. It then follows that the energy of the gravitational radiation associated with each photon must similarly be quantised, as already mentioned on Section 2. The new term introduced in this paper in the above text "gravitation quantum” is therefore physically justified.

Therefore it is assumed that Planck's equation for the energy of the high-frequency gravitational radiation associated with the photon $(E=h \cdot v)$ could also apply to cosmic low-frequency gravitational waves, should they actually exist (because two differing formulas are not possible for one physical phenomenon). If that were the case, then cosmic gravitational waves would also have Maxwell's theory as a physical foundation rather than Einstein`s General Relativity theory. And if that were true, cosmic gravitational waves could not be "perturbations of space-time”, but would have to expand in exactly the same manner as electromagnetic waves. This may possibly be the reason that it had not been possible to detect cosmic gravitational waves up to now.

Remark: In his book "The Structure of Physics" (Aufbau der Physik), C. F. von Weizsäcker [4] determined that all information relating to natural sciences-and particularly energy $\equiv$ matter-is derived from captured (registered) photons. Every sensory perception is associated —in an intermediate step as a minimum —with photons. In other words, there can be no perception (information) without the electromagnetic interaction (photons). The dimensionless variable "information" is therefore the most fundamental variable in physics.

Due to the quantum nature of light, it further follows that all physical information is digital! From this C. F. von Weizsäcker draws the following conclusion: If the whole of physics is based on quantised information, then similarly, the whole of physics can only consist of quantised variables. (This statement of C. F. von Weizsäcker also confirms that the hitherto unknown term "gravitational quantum” introduced here is perfectly justifiable). 


\section{Unification of Gravitation and Electromagnetism}

A photon's electromagnetic radiation forms a physical dualism with the gravitational radiation associated with it (complete equality of both components linked to each other) on the common basis of the same Maxwellian theory. It is not therefore surprising that the equation $\mathrm{E}=$ $h \cdot v$ applies equally both to the energy of the electromagnetic radiation and the high-frequency gravitational radiation associated with photons.

Although static gravitation is the weakest of the four fundamental forces in the universe, it is surprising to discover here that:

At the speed of light, the intrinsic gravitation of a photon as gravitational radiation or dynamic gravitation is selectively (related to its photon) just as strong as the electromagnetic radiation (of the photon), but acting in an opposing manner, so that both of these fundamental forces exist in a stable equilibrium and cancel each other out from an external perspective. Electromagnetism and its dynamic gravitation are perfectly united and unified in each photon!

Each photon carries with it its exactly specific gravitation quantum. Each photon forms an opposite interacting pair of forces with its gravitation quantum (precisely stated: they are mediators of two opposite interacting forces), which exists in a stable equilibrium precisely at the speed of light.

In the micro-world of photons, their dynamic gravitation is unambiguously a force and not a geometry (otherwise they couldn't get unified with electromagnetism), which at the speed of light precisely opposes the electromagnetic force and selectively "neutralises” it!

Remark: In Einstein's Theory of General Relativity (1915), static gravitation is not a force but a property of space-time under the influence of cosmic (baryonic) masses, which is what corresponds to the geometry of the cosmos. On the other hand, there is no doubt that gravitation (or the force of gravity) is one of the four fundamental forces of the universe that causally determine the geometry of the cosmos and its structures. Gravitation is not, however, the geometry of the cosmos in itself; the geometry is rather an effect of the static gravitation of the cosmic agglomeration of masses (including the nonbaryonic dark matter, which remained unknown until the 1960s). If cause and effect are interchanged, then this can result in a mistaken perception of reality!

If the internal relationship between the photons' intrinsic gravitation and the speed of light described in these pages is correct, then at the speed of light the selective gravitational radiation of photons is only a mirror image and hence a facet of the electromagnetic radiation; thus the same vectorial Maxwellian theory applies in both cases!
“Albert Einstein is supposed to have spent 20 years attempting to describe electromagnetic interaction and gravitation as two aspects of a single higher-level interaction. All to no avail-because even today it is still not known whether it is possible to unify gravitation with other interactions” [5].

"Today's physicists are largely convinced that gravitation plays a key role not only in cosmology but in elementary particle physics as well (particularly applicable in the case of the photon), but this role is currently not yet properly understood" [5].

The considerations provided in this paper may possibly be of help in the hitherto unsuccessful search for a theory unifying gravitation with electromagnetism, because, at the level of photons travelling at the speed of light, a complete union and unification of these fundamental forces of nature, reduced to the "common basis $\mathrm{E}$ $=h \cdot v$ ” take place from a physical perspective. The union of gravitation with electromagnetism is also already clearly expressed in the equation $\mathrm{G} / \mathrm{c}^{2}=$ const. respectively $\mathrm{G} \cdot \varepsilon_{0} \cdot \mu_{0}=$ const.

\section{Law of Conservation of Gravitation}

A photon can never be made "gravity-free" (if this were possible it would no longer be a photon), because intrinsic gravity is a fundamental component of each photon just as of any other form of energy. As is the case for the photon itself, its gravitation quantum does not possess any baryonic rest mass, it is electrically neutral and cannot like energy be annihilated; it remains in existence forever, having a virtually unlimited lifetime. It therefore follows that in the event of energy transformation there must also exist - analogous to the "Law of Conservation of Energy"-a "Law of Conservation of Gravitation", which "expressis verbis" appears neither in my physics books nor in any cosmological book available to me, and which I have formulated as follows:

For every transformation of energy, the gravitation quantum corresponding to the converted amount of energy in each case is also transferred, so that the effect of the original gravitation quantum existing before the energy transformation is conserved overall. This conservation principle possesses the character of a law of nature, and it may possibly be the first time it has been mentioned.

As the cosmos at the time zero in the cosmic calendar contained a finite large and constant amount of energy ("pure" radiation energy, i.e. photons having the highest energy level in the history of creation), with this energy being subject to an ongoing transformation process, then the gravitation quantum appearing at the same time asand associated with - this amount of energy must also be constant and remain conserved overall for the entire duration of existence. Therefore gravitation, as is the case 
with energy, can be neither created nor annihilated, thus also pointing to the same origin as energy at the same instant.

\section{Conclusions}

It is possible to derive the relationship $\mathrm{G} / \mathrm{c}^{2}=$ const. from the Gravitational Constant $G$ itself; this infers that $c$ would not actually be an "independent" universal constant of nature, but dependent on $\mathrm{G}$ and therefore not a "genuine" constant of nature. The expression $\mathrm{G} / \mathrm{c}^{2}=$ const., on the other hand, could well be both a "genuine" and "united" constant of nature, applicable as far back as the quantum era, because $\mathrm{G} / \mathrm{c}^{2}=\mathrm{l}_{\text {Planck }} / \mathrm{m}_{\text {Planck }}=$ $\mathrm{G}_{\text {super }} / \mathrm{c}_{\text {super }}^{2}=\mathrm{G} \cdot \varepsilon_{0} \cdot \mu_{0}=$ const. $(\mathrm{m} / \mathrm{kg})$

The key insight derived in this paper is the fact that electromagnetism - and thus also photons-are associated with a hitherto unnoticed or unknown dynamic gravitation, equally strong as electromagnetism but acting with opposite effect, which has to be included in all relevant physical considerations. This gravitation of photons is therefore not static in nature, as previously assumed, but just as dynamic as the electromagnetic energy of photons. This consequently results in the now following new insights:

Each photon as a quantum of electromagnetic energy is closely connected with an equivalent quantum of gravitational energy. Due to its intimate meshing with the photon or its associated electromagnetic photon radiation, this gravitational quantum itself also appears as radiation, namely as specific high-frequency gravitational radiation bound to the photon. Therefore each photon consists of two components linked to each other, one expansive electromagnetic radiation component and one gravitational radiation component acting in an equal and opposite manner. Therefore there must exist exactly the same number of gravitational quanta of the photons as of photons themselves.

The speed of light $\mathrm{c}$ in a vacuum is therefore a constant $299,792,458 \mathrm{~m} / \mathrm{secs}$, since this is exactly the value, at which a stable state of equilibrium is established between the dynamic gravitation force of the photons and their expansive electromagnetic force, the two opposing forces thus cancelling each other out and resulting in a total zero energy of all photons.

The constancy of speed of light is achieved by a "physical regulatory mechanism". As a "regulator" for the constancy of the speed of light the only candidate is thus the intrinsic gravitation of the photons themselves, since any change in energy of a photon simultaneously causes an equal change in its intrinsic gravitation. Therefore the speed of light remains constant for all possible energy situations of a photon.

The physical principles of both the photon's electromagnetic radiation and its high-frequency gravitational radiation must be in every respect completely equivalent but opposed to each other. It therefore follows that the same vectorial Maxwellian theory also underlies both physical quantities, i.e. they also possess a common theoretical basis!

Planck's equation for the energy of the electromagnetic radiation of a photon $\mathrm{E}=\mathrm{h} \cdot v$ must also apply to the high-frequency gravitational radiation, which is associated with the photon and only selectively acts on it. It then follows that the energy of the gravitational radiation associated with each photon must similarly be quantised.

A photon's electromagnetic radiation forms a physical dualism with the gravitational radiation associated with it on the common basis of the same Maxwellian theory. Electromagnetism and its dynamic gravitation are perfectly united and unified in each photon!

Analogous to the "Law of Conservation of Energy" a "Law of Conservation of Gravitation" must also exist.

It should be noted that the dynamic gravitation of photons and their conversion into static gravitation following the energy transformation of photons may provide a plausible explanation for the mysterious "dark matter" and "dark energy". In view of the enormous significance of the "dark phenomena of the cosmos" for our world view from a natural science perspective, I have written a separate paper titled: "The hunt for dark matter and dark energy".

\section{REFERENCES}

[1] K. O. Greulich, "The Universality of the Specific Planck Charge and Its Role for Gravitation,” Lecture Presented at the German Physical Society, Dresden, 2011.

[2] W. Thirring, "Baupläne der Schöpfung-Hat Die Welteinen Architekten? The Building Plans of Creation-Does the World Have an Architect?” Seifert Publishing House, Vienna, 2011, pp. 36-37.

[3] K. O. Greulich, "Constant Constants since the Big Bang?" Lecture Presented at the German Physical Society, Göttingen, 27 February-3 March 2012.

[4] C. F. von Weizsäcker and A. der Physik, "The Structure of Physics,” Deutscher Taschenbuch Verlag, München, 2002.

[5] B. Povh, "Elementary Particle Physics and Cosmology," Sterne und Weltraum, No. 3, 2004, pp. 32-35. 